\title{
High Temperature Endurable Fiber Optic Accelerometer
}

\author{
Yeon-Gwan Lee, ${ }^{1}$ Jin-Hyuk Kim, ${ }^{2}$ and Chun-Gon Kim ${ }^{2}$ \\ ${ }^{1}$ The 1st R and D Institute 2, Agency for Defense Development, P.O. Box 35, Yuseong, Daejeon 305-600, Republic of Korea \\ ${ }^{2}$ Department of Aerospace Engineering, Korea Advanced Institute of Science and Technology, 291 Daehak-ro, Yuseong-gu, \\ Daejeon 305-701, Republic of Korea
}

Correspondence should be addressed to Chun-Gon Kim; cgkim@kaist.ac.kr

Received 25 January 2013; Accepted 27 April 2013; Published 12 March 2014

Academic Editor: Gyuhae Park

Copyright (C) 2014 Yeon-Gwan Lee et al. This is an open access article distributed under the Creative Commons Attribution License, which permits unrestricted use, distribution, and reproduction in any medium, provided the original work is properly cited.

\begin{abstract}
This paper presents a low frequency fiber optic accelerometer for application in high temperature environments of civil engineering structures. The reflection-based extrinsic fiber optic accelerometer developed in this study consists of a transmissive grating panel, reflective mirror, and two optical fiber collimators as the transceiver whose function can be maintained up to $130^{\circ} \mathrm{C}$. The dynamic characteristics of the sensor probe were investigated and the correlation between the natural frequency of the sensor probe and temperature variation was described and discussed. Furthermore, high temperature simulation equipment was designed for the verification test setup of the developed accelerometer for high temperature. This study was limited to consideration of $130^{\circ} \mathrm{C}$ applied temperature to the proposed fiber optic accelerometer due to an operational temperature limitation of commercial optical fiber collimator. The sinusoidal low frequency accelerations measured from the developed fiber optic accelerometer at $130^{\circ} \mathrm{C}$ demonstrated good agreement with that of an MEMS accelerometer measured at room temperature. The developed fiber optic accelerometer can be used in frequency ranges below $5.1 \mathrm{~Hz}$ up to $130^{\circ} \mathrm{C}$ with a margin of error that is less than $10 \%$ and a high sensitivity of $0.18\left(\mathrm{~m} / \mathrm{s}^{2}\right) / \mathrm{rad}$.
\end{abstract}

\section{Introduction}

Over the past three decades, many types of fiber optic accelerometers (FOAs) have been developed because optical fibers (OFs) allow structural health monitoring within highly electromagnetic environments [1].Furthermore, optical fiber with fused silica is useful in a wide temperature range up to about $1000^{\circ} \mathrm{C}$ [2] although it depends on the optical fiber material such as fused silica and sapphire $\left(\mathrm{Al}_{2} \mathrm{O}_{3}\right.$, single crystal alumina) $[3,4]$.

While most intensity-based fiber optic sensor systems are relatively low cost, fiber Bragg grating (FBG) sensor systems are rather higher cost wavelength shift interrogation equipment for high speed sampling frequency rates. Alternatively, intensity modulation techniques have been conducted on grating-based extrinsic type fiber optic sensors (FOSs) [5-7] because the grating-based sensors [8,9] possess significant advantages [10]: simple mechanical structure and good reliability, among others.

Therefore, research on grating-based fiber optic sensors has been conducted based on the shutter effect $[9,11]$ or the Moiré phenomenon techniques [7], which require four optical fiber lines and two grating panels. However, these transmission type FOSs $[6,7,9,11]$, including the Moiré fringe-based FOA, depending on the transmitted light between the separated optical fibers, require the placement of optical fibers (OFs) on both sides of the prototype [69 , 11]. Consequently, this leads to significant limitations during corner installations due to the geometric conditions. When the FOAs are used to monitor large structures using multipoint sensors in hazardous locations, the issues of complex cabling are further intensified.

Nowadays, in order to resolve these problems, reflectionbased techniques using grating panel have been developed $[12,13]$. This resolved the difficulties in corner installations and complex cabling problems in multipoint sensing due to the $50 \%$ simplified cabling by employing collimated optical fibers as transceivers, which allows the fibers to be placed on only one side of the sensor case. However, this FOA was reported to withstand temperatures up to only $65^{\circ} \mathrm{C}$ due to the use of epoxy for assembly. Thus, most reported FOAs have been limited to handling temperatures up to 
$60^{\circ} \mathrm{C}$ although many kinds of civil engineering structures are exposed to high temperature environments. For instance, for ambient vibration monitoring of a pipe in nuclear power plant structures where hot water after cooling flows through, high temperature resistant and temperature independent materials must be used for sensor fabrication. Except for various kinds of fiber-based temperature sensors, which are able to operate beyond $1000^{\circ} \mathrm{C}[3,4]$, and pressure sensors [14] for high temperature environments, there are almost no low frequency FOAs available for use in high temperature environments in civil engineering structures. This is because sensor structure assemblies usually involve numerous materials including silicon, glass with a low softening point, polymer, and epoxy as an adhesive. As a result, the thermal expansion mismatch among these materials can lead to stability degradation of the sensors' accuracy, resolution, and repeatability [15].

Therefore, as a fundamental study, this paper presents an FOA that uses a reflection-based technique using a grating panel for application to high temperature environments in civil engineering structures such as a plumbing pipe, in which hot water flows through, of nuclear power plant structures. The targeted operating temperature is $130^{\circ} \mathrm{C}$ due to the operational temperature limitation of the commercial optical fiber collimator. The fiber optic accelerometer that can endure $130^{\circ} \mathrm{C}$ was fabricated using mostly the following materials: invar, ceramic adhesive, and $130^{\circ} \mathrm{C}$ endurable collimated optical fiber for dimensional stability in high temperature environments. Furthermore, a high temperature adjustable chamber was developed and installed with a shaker for the accelerometer performance test. The dynamic characteristics of the sensor probe were investigated after a prototype was fabricated. The correlation between the natural frequency of the sensor probe and temperature variation was empirically investigated. Then a performance test of the $130^{\circ} \mathrm{C}$ endurable FOA was implemented through a comparison with a commercial low frequency accelerometer.

\section{Working Principles}

In this study, the foundational quantification principle of acceleration states, in which the change in acceleration is proportional to the change in the relative displacement of a seismic mass, was considered. Moreover, the acceleration can be calculated using the measured relative displacement of the seismic mass through

$$
\ddot{u}_{0}=u(t) \cdot \sqrt{\left(1-r^{2}\right)^{2}+(2 \zeta r)^{2}} \cdot \omega_{n}^{2},
$$

where $r=\left(\omega / \omega_{n}\right), \zeta$ is the damping ratio, $\omega_{n}$ is the natural frequency of the integrated mass-spring system, and $\ddot{u}_{0}$ is the acceleration imparted on the seismic mass.

In order to convert the relative displacement into acceleration, the natural frequency and damping ratio of the sensor probe need to be identified. The sensor head as a single degree of freedom structure consists of a single transmissive grating panel (TGP), a reflective mirror, and two transceiver OFs that are separated by a distance of a quarter of the grating pitch $l$ to have a phase difference of $90^{\circ}$ between the two received signals $[12,13]$, as shown in Figure 1 , in order to obtain the relative displacement of the seismic mass. The variable $u_{1}$ refers to the seismic mass displacement and the variable $u_{0}$ refers to the sensor case displacement. If the periodic sine wave signals are obtained in quadrature, the relative displacement $u$, defined as the difference of $u_{1}$ and $u_{0}$, can be inferred by unwrapping the two normalized received signals and can be expressed using sine and cosine functions, as follows [16]:

$$
u(t)=\frac{l}{2 \pi} \cdot \text { unwrap }\left[\tan ^{-1}\left\{\frac{(\sin (2 \pi \cdot u(t) / l))}{(\cos (2 \pi \cdot u(t) / l))}\right\}\right] .
$$

The TGP consists of multiple opaque gratings with evenly spaced transparent spacings. The opaque grating width $\left(l_{1}\right)$ and transparent spacing $\left(l_{2}\right)$ are smaller than the optical beam width. The grating pitch of $l$ is the additive sum of $l_{1}$ and $l_{2}$. The OFs and reflective mirror, placed on the back of the TGP and perfectly aligned to the end surfaces of the OFs, are fixed to the sensor case in order to produce stable reflectivity.

When the excited acceleration is assumed to be defined as $\ddot{u}_{0}=A_{\text {exc }} \cdot e^{i \omega t}$ where $\omega$ is the excited frequency, the steady state response should be the relative displacement of the mass defined as $u=B_{\text {res }} \cdot e^{i \omega t}$. $A_{\text {exc }}$ refers to the acceleration measured through the reference accelerometer and $B_{\text {res }}$ refers to the seismic mass measured displacement in the FOA. Therefore, through the deformation response factor $R$, the acceleration can be obtained theoretically, which is expressed using the relationship between the excited acceleration and relative displacement, as defined by the following equation:

$$
R=\left|\frac{B_{\mathrm{res}}}{A_{\mathrm{exc}}}\right| \cdot \omega_{n}^{2}=\frac{1}{\sqrt{\left(1-r^{2}\right)^{2}+(2 \zeta r)^{2}}},
$$

where $r=\omega / \omega_{n}, \zeta$ is the damping ratio, and $\omega_{n}$ is the natural frequency of the integrated mass-spring system. The quantification of the parameters such as the relative displacement and acceleration was accomplished using this mechanism.

\section{Fabrication of a Prototype for High Temperature Environments}

In order to develop the $130^{\circ} \mathrm{C}$ endurable FOA, the basic material of the prototype for dimensional stability, the adhesive for the assembly of the sensor components, and the fiber optic collimator that has to maintain its function as a collimator at $130^{\circ} \mathrm{C}$ were considered.

3.1. Fabrication of Sensor Probe. In order to realize the sensor principles at high temperature, the parallel alignment between the reflective surface of the mirror and the end surface of the collimated optical fiber should be maintained during operation at the targeted high temperature. To comply with this requirement, the dimensional stability and consistent functionality of the sensor components such as the collimator and TGP were important consideration factors. Basically, the sensor probe was designed and fabricated based on the researched stable sensor probe without miniscule 


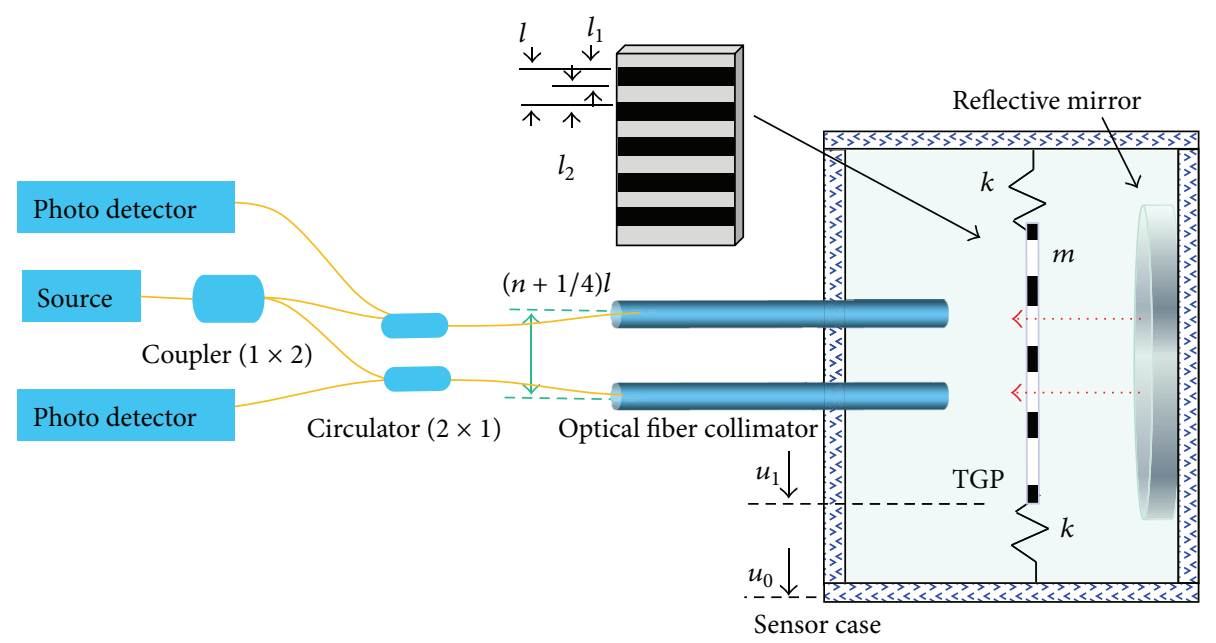

FIGURE 1: Schematic diagram of the transmissive grating-reflective mirror based FOA sensor system [12].
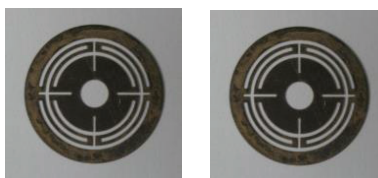

〈Two leaf springs〉

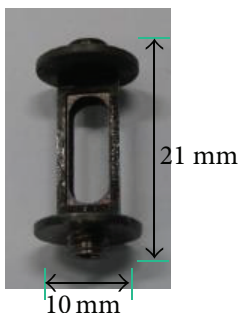

$10 \mathrm{~mm}$

〈Seismic mass〉

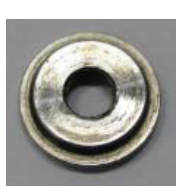

$\langle$ Clamping tool for spring 〉
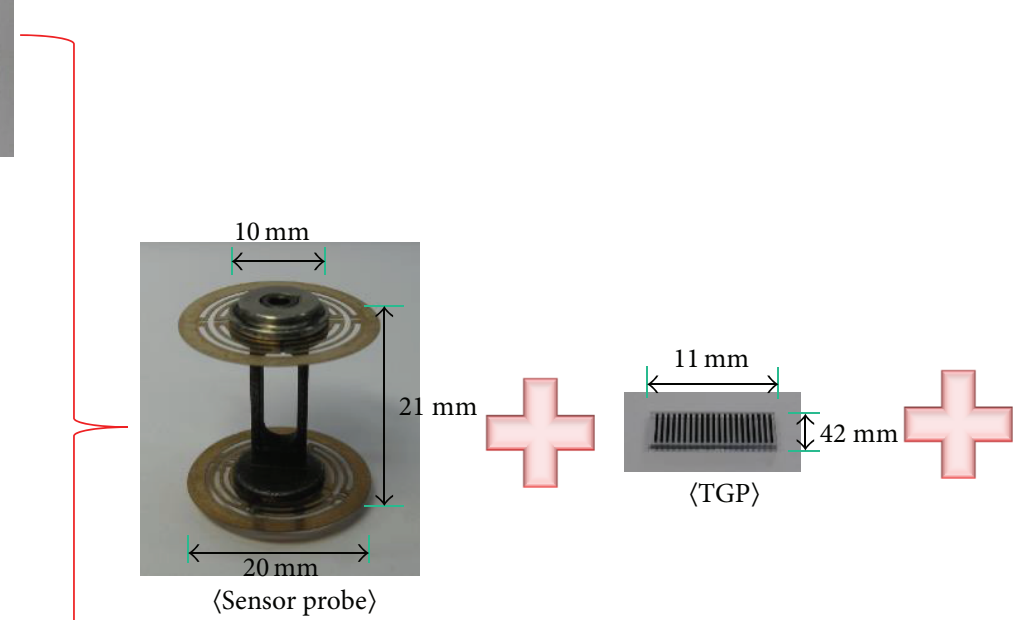

$\langle$ TGP $\rangle$

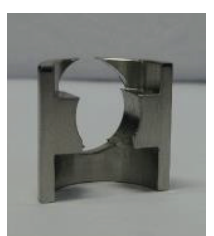

$\langle$ Mirror supporter $\rangle$

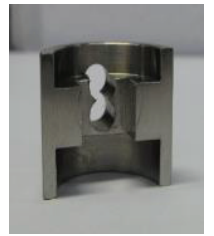

〈OF supporter〉

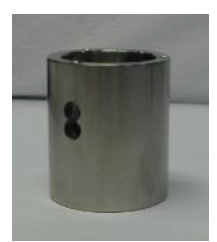

$\langle$ Case $\rangle$

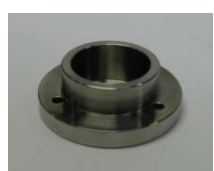

$\langle$ Tap $\rangle$

FIGURE 2: Key components for a $130^{\circ} \mathrm{C}$ endurable FOA with an invar base.

rotation and lateral motion of the seismic mass [17]. For seismic motion, a leaf plate with a pattern was employed as a spring. The leaf spring is composed of phosphor bronze with a thickness of $60 \mu \mathrm{m}$. These are placed on both the top and bottom of the sensor probe with horizontal symmetry, as shown in Figure 2. The circular clamping tools were used to fix the leaf spring on the seismic mass. First of all, clamping tools, which have the possibility of causing distortion of the leaf springs at an elevated temperature, were made of invar material due to its low coefficient of thermal expansion (CTE, $\left.1.2 \times 10^{-6} / \mathrm{K}\right)$. Furthermore, in order to prevent distortion of the TGP due to CTE difference between the TGP, whose base material is quartz, and the seismic mass, the seismic mass was made using invar material because the TGP has to be attached to the seismic mass. The sensor probe, which plays a pivotal role in the accelerometer, was constructed by 
assembling two fabricated leaf springs and clamping tools. In addition, the mirror supporter, OF supporter, and sensor case were also fabricated using invar material because the optical fiber collimators and mirror have to be supported and fixed by them. Therefore, in order to keep their alignment at high temperature, dimensional stability among them must be guaranteed. Consequently, all sensor components were made with an invar base. The sensor probe had a size of 20 (outer diameter of spring) $\times 21$ (height) $\mathrm{mm}^{2}$. The TGP (thickness: $700 \mu \mathrm{m}$ ), which has a size of $11.0 \times 4.2 \mathrm{~mm}^{2}$, was employed to be attached to the center of the fabricated seismic mass. Furthermore, a ceramic adhesive (940LE, Cotronics Co., USA), whose strength can be maintained up to $1357^{\circ} \mathrm{C}$, was used for the assembly of all the optical components for dimensional stability and high adhesive strength.

3.2. FOA Prototype. In this research, a pigtail-type fiber optic collimator is used to minimize the emitted light dispersion at the end surface of the OF and maximize the reflected light receiving rate from the reflective mirror. Most commercial pigtail-type collimators, which employ ferrule and grin lenses, are not designed to withstand high temperatures in terms of dimensional stability and adhesive strength of the bonding material used and the polymer jacket, which was used to avoid damage of the optical fiber line. The dimensional instability may cause difficulties in holding the alignment between the end of the optical fiber and the lens at elevated temperatures. Therefore, commercial fiber optic collimators were customized in order to withstand temperatures up to $130^{\circ} \mathrm{C}$ by OZ Optics Corporation. Two customized single mode optical fibers combined with the collimator (LPC-06-1550-9/125-S-0.5-2.61CL-40-3S-1-5-HTSP, OZ Optics Co., Canada) were bonded to the sensor case using ceramic adhesive, as shown in Figure 3. The TGP, which has an $l$ of $280 \mu \mathrm{m}, l_{1}$ (Cr material) of $140 \mu \mathrm{m}$, and $l_{2}$ of $140 \mu \mathrm{m}$, was also attached to the center of the sensor probe using ceramic adhesive. The reflective aluminum mirror (PF05-03-G01, Thorlabs, Inc., USA) was also fixed to the sensor case on the opposite side of the optical fiber. The FOA prototype was assembled and it had dimensions of 30 (outer diameter) $\times 26$ (height) $\mathrm{mm}^{2}$ and a total weight of $95.7 \mathrm{~g}$. Although it is relatively large in comparison to the MEMS type low frequency accelerometer, the FOA prototype was manufactured to be similar in size to the PZT-based or servo type low frequency accelerometers.

\section{4. $130^{\circ} \mathrm{C}$ Endurable Fiber Optic Accelerometer}

4.1. Dynamic Characteristics of the Sensor Probe according to the Temperature Variations. The mechanical properties of most materials are affected by temperature variations. Young's modulus of phosphor bronze, which was used for the leaf springs, decreases as the applied temperature increases [18]. This indicates that the spring coefficient of the $m-k$ structure leads to a change in the natural frequency of the sensor probe at elevated temperatures. To verify the sensor performance regarding the effect of temperature, the natural frequency variations of the sensor were investigated. When the sensor

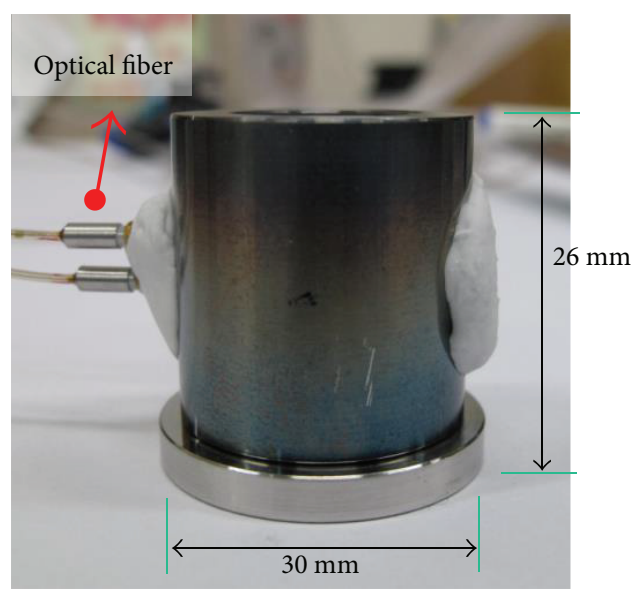

Figure 3: Prototype of $130^{\circ} \mathrm{C}$ endurable FOA.

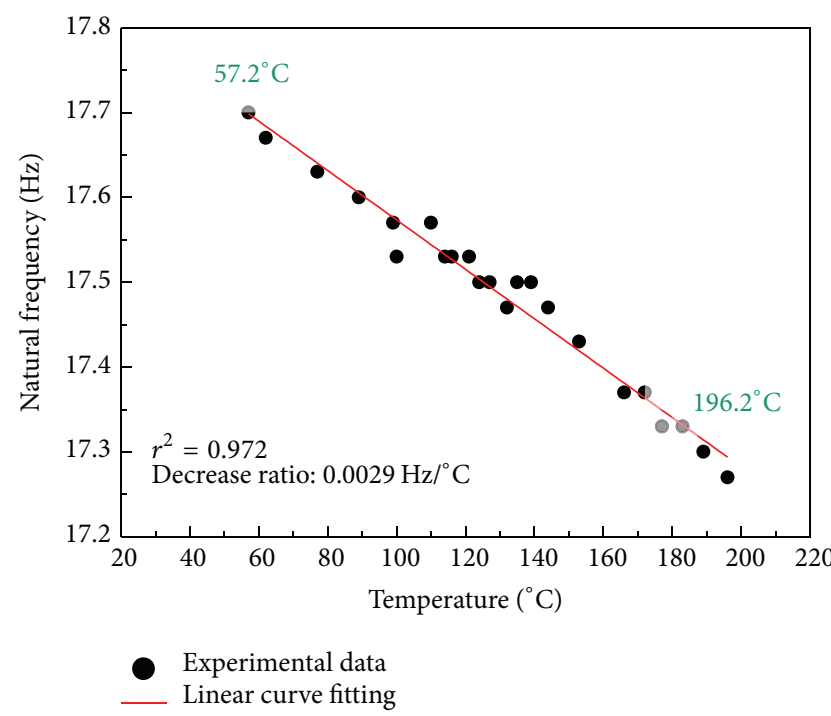

FIGURE 4: 1st natural frequency variations of the sensor probe according to the surrounding temperature variations.

was used at the equilibrium state of the elevated temperature, the measurable frequency range was found to be affected by the temperature variation. At this equilibrium state, the natural frequencies of the sensor obtained experimentally are plotted according to the corresponding equilibrium temperatures, and, therefore, the measurable frequency range of the FOA can be estimated according to the change in temperature, as shown in Figure 4.

The sensor probe without the TGP and bonding material was excited by an impact hammer, and then the impulse response function was measured by the laser displacement sensor (LK-H050, Keyence Co., Japan). A natural frequency of $17.77 \mathrm{~Hz}$ at $28^{\circ} \mathrm{C}$ and a damping ratio of 0.00073 were empirically achieved from the free vibration test. The weight of the sensor probe with seismic mass, two leaf springs, and two clamping tools was measured to be $4.27 \mathrm{~g}$.

The natural frequency of the sensor probe is an important performance factor because the measurable frequency range of the FOA depends on the natural frequency of 


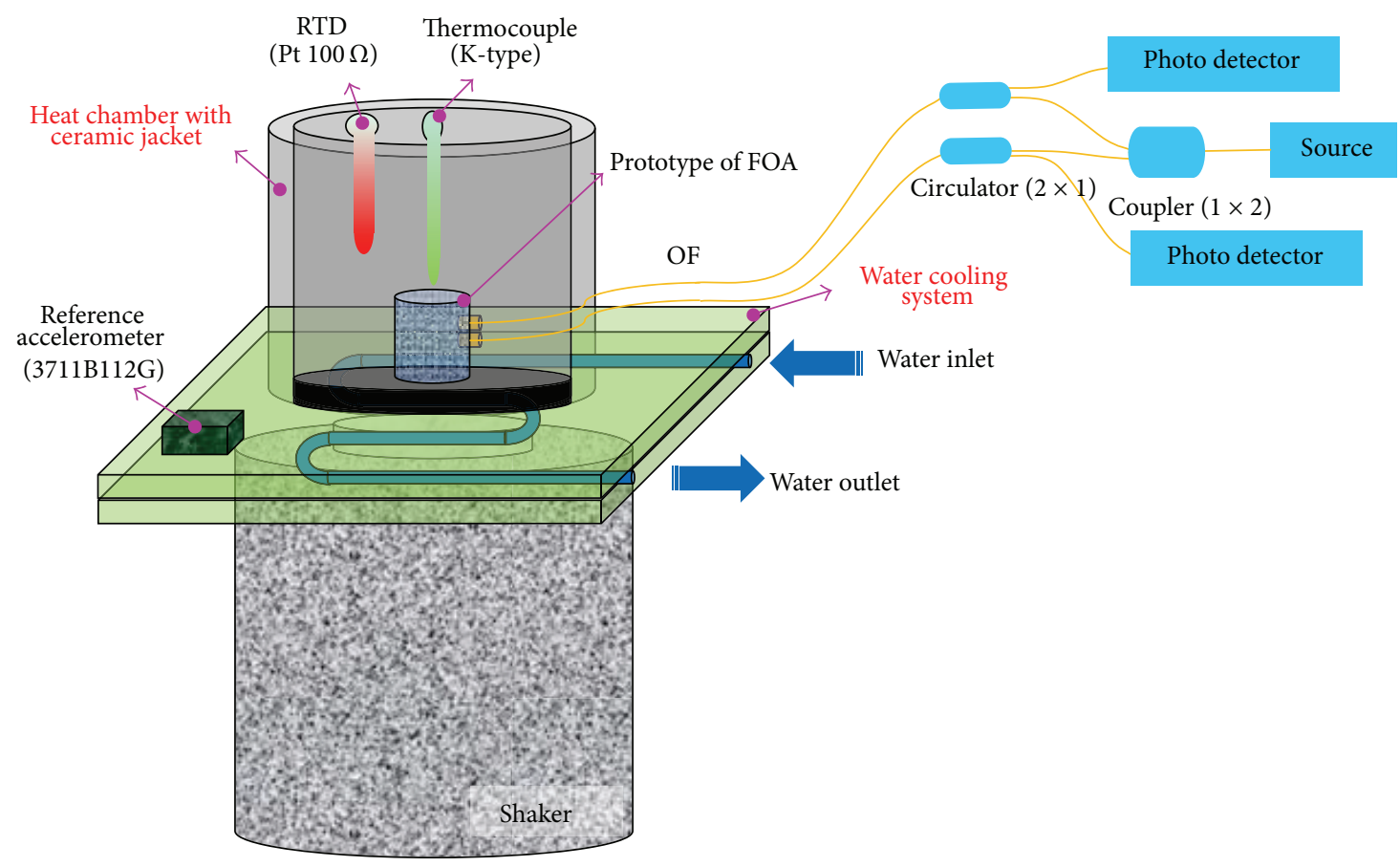

FIGURE 5: Experimental setup for the performance test of the FOA.

the embedded sensor probe. Most accelerometers can be used below one-third of the natural frequency. In the temperatureequilibrium state, the correlation between the natural frequency of the sensor probe and temperature variation was investigated, as shown in Figure 4. The sensor performance regarding sensitivity and the measurable acceleration range among others also depends on the spring coefficient. Therefore, the experiment for the temperature effect on the sensor was limited to the consideration of the natural frequency variation of the sensor.

The temperatures applied to the sensor were set to vary from $57.2^{\circ} \mathrm{C}$ to $196.2^{\circ} \mathrm{C}$. In the linear regression analysis below the temperature of $196.2^{\circ} \mathrm{C}$, the $R$-square value between the natural frequency and temperature was 0.972 while the decrease ratio of the natural frequency was approximately $0.0029 \mathrm{~Hz} /{ }^{\circ} \mathrm{C}$. This indicates that the natural frequency of the sensor linearly decreased with an increase in the sensor temperature. The decrease ratio can be altered by the level of the maximum experienced temperature because the mechanical property variation of the phosphor bronze depends on the maximum experienced temperature. Thus, due to a small decrease in natural frequency, the fabricated sensor probe is expected to measure acceleration without large variation of the measurable frequency range.

The result of natural frequency reduction with an increase in temperature indicates that the spring coefficient may be reduced due to a decrease in Young's modulus of the phosphor bronze (spring material). In addition, after annealing at a nonexperienced temperature, the natural frequency can be changed at room temperature due to the mechanical property change of the leaf spring.
4.2. Experimental Setup. In order to verify the performance of the fabricated FOA according to temperature variations, the use of an exciter that is stabilized against temperature variations and a large temperature chamber that the exciter can be placed inside is necessary. However, it may be practically difficult to set up a temperature variable acceleration test system because it would require all components, accessories, and equipment, such as the shaker and reference accelerometer, to satisfy a certain temperature resistance (dimensional stability) necessary for the validity of the test. In addition, a large temperature chamber may also be required. Therefore, a simple and new installation for verification was fabricated, as shown in Figure 5. A K-type thermocouple was employed to control the temperature of the chamber. Accurate temperature of the chamber inside can be measured using a resistance temperature detector (RTD) with a platinum $100 \Omega$. The fabricated FOA was placed inside the chamber where the environmental temperature can be controlled by a ceramic jacket. Consequently, the FOA prototype and the microelectromechanical system (MEMS) reference sensor (3711B112G, PCB Piezotronics, Inc., USA), which has a high sensitivity of $101.9 \mathrm{mV} /\left(\mathrm{m} / \mathrm{s}^{2}\right)$ at low frequency acceleration, were fixed to the shaker. The reference accelerometer and the fabricated $130^{\circ} \mathrm{C}$ endurable FOA were placed on the shaker (PM50A, MB Dynamics Co., OH, USA), whose motion was controlled using a sine function generator. However, the commercial accelerometer and the shaker have to be protected from heat transfer because they are not guaranteed for use at high temperature. Therefore, a water cooling system in plate form was installed between the heat chamber and the shaker. While heat was applied to the chamber inside by the ceramic jacket, cold running water was used as a 


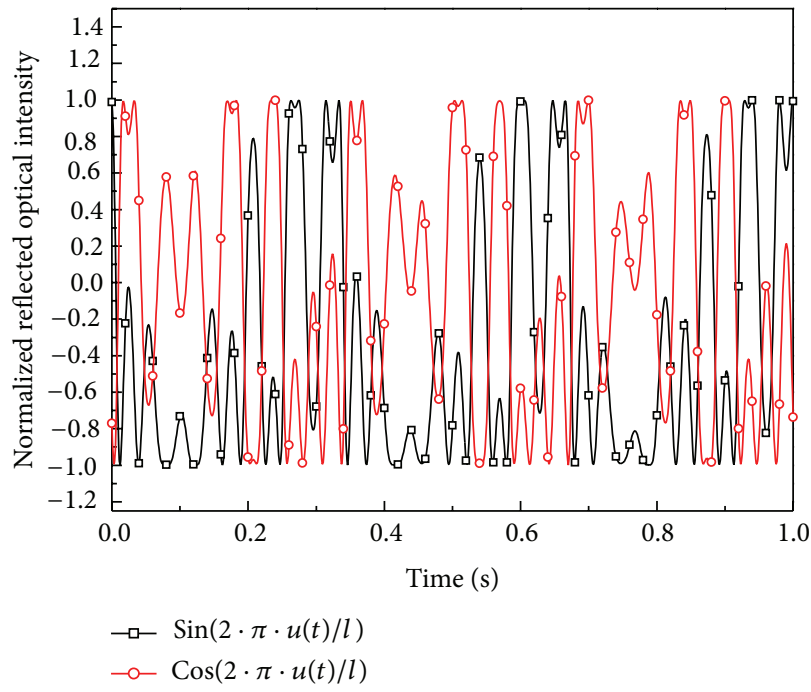

(a) Two normalized reflected optical signals in quadrature at $3.0 \mathrm{~Hz}$ excitation.

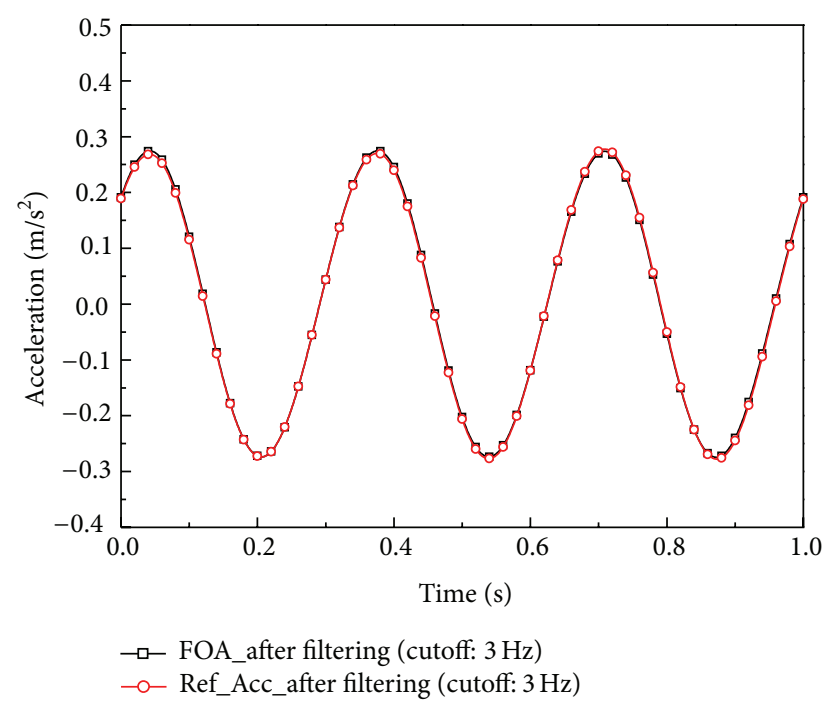

(b) Comparison of the acceleration measured using the proposed FOS $\left(130^{\circ} \mathrm{C}\right)$ and a commercial reference accelerometer $\left(24^{\circ} \mathrm{C}\right)$ at $3.0 \mathrm{~Hz}$ excitation with low pass filtering of $3.0 \mathrm{~Hz}$.

Figure 6: Measured normalized optical signals and acceleration at $130^{\circ} \mathrm{C}$.

coolant. Furthermore, the reference accelerometer was placed on the edge of the water cooling plate where the heat cannot be transferred. During the experiment, the temperature was maintained at room temperature without heat transfer to the reference sensor. Therefore, the reference acceleration data was obtained at room temperature where the acquired data was reliable.

An erbium doped fiber amplifier (EDFA; $1530-1565 \mathrm{~nm}$, JDS Uniphase Co., USA) was used as the light source. As the plate that was fixed to the shaker was excited, the received lights of the $130^{\circ} \mathrm{C}$ endurable FOA were stored in the data acquisition (DAQ) system at sampling rates of $1 \mathrm{kHz}$ through the two circulators (PICT-1550-S, Oyokoden Lab., Tokyo, Japan) and photo detectors (v2011, New Focus, Inc., USA).

4.3. Performance of the Fiber Optic Accelerometer. In the fabricated prototype, the weight of the sensor probe increased after bonding the TGP with ceramic adhesive. The initial natural frequency before annealing was $17.27 \mathrm{~Hz}$ with a standard deviation of 0.03 . The natural frequency after annealing at $130^{\circ} \mathrm{C}$ was $17.09 \mathrm{~Hz}$ with a standard deviation of 0.06 at $24.6^{\circ} \mathrm{C}$ due to mechanical property change. The natural frequency of $16.91 \mathrm{~Hz}$ at $130^{\circ} \mathrm{C}$ was experimentally determined. $1.04 \%$ of the initial natural frequency decreased until an elevated equilibrium temperature of $130^{\circ} \mathrm{C}$ was reached. The natural frequency of the sensor probe changed because of the increased weight of $0.25 \mathrm{~g}$ due to the added TGP and ceramic bonding material used between the TGP and seismic mass. As the shaker vibrated the base plate, the reflected signals at the two observation points were periodic sine wave functions of the relative displacement $l$ with a phase difference of $90^{\circ}$ with respect to each other. The raw reflected signals of these two points can be expressed as a DC component. The two normalized reflected signals were plotted after normalization to a sinusoidal function, which has an amplitude of 1 and a mean magnitude of 0 , as shown in Figure 6(a). This figure describes the normalized reflected optical signals of fiber optic collimator $1(\mathrm{OF} 1)$ and fiber optic collimator 2 (OF2) at the $3.0 \mathrm{~Hz}$ excitation. Figure 6(b) shows the comparison of the acceleration measured using the proposed FOA and a commercial reference accelerometer at $3.0 \mathrm{~Hz}$ excitation with low pass filtering of $3.0 \mathrm{~Hz}$. The measured acceleration data of the developed FOA and reference accelerometer were tracked at $130^{\circ} \mathrm{C}$ and $24^{\circ} \mathrm{C}$, respectively. The measured root mean square (RMS) values of the vibrated acceleration were approximately $0.195 \mathrm{~m} / \mathrm{s}^{2}$ at $3.0 \mathrm{~Hz}$. At $3.0 \mathrm{~Hz}$ excitation, the acceleration measured by the proposed FOA agrees very well with that of Ref_Acc after filtering. This indicates that the fabricated FOA including the OF line with a jacket operates well at $130^{\circ} \mathrm{C}$.

The measured relative displacement of the seismic mass was $49.37 \mu \mathrm{m}$ (peak-to-peak magnitude). The acquired voltage from the normalization signal processing was $1.11 \mathrm{rad}$ because the acquired period of the sinusoidal signal was 0.18 periods. The average sensitivity was $5.68 \mathrm{rad} /\left(\mathrm{m} / \mathrm{s}^{2}\right)$ based on the RMS acceleration value criterion because the magnitude of the measured RMS acceleration value was $0.20 \mathrm{~m} / \mathrm{s}^{2}$ (peakto-peak magnitude) after low pass filtering. This indicates that the proposed FOA has a higher sensitivity of $55.69 \mathrm{rad} /[\mathrm{G}]$ (gravitational acceleration $\mathrm{G}$ ). The minimum measurable acceleration of the fabricated prototype was $0.0016 \mathrm{~m} / \mathrm{s}^{2}$ based on the RMS acceleration value criterion because of a minimum measurable displacement of $0.41 \mu \mathrm{m}$ of the sensor probe.

The frequency response curve of the FOA was experimentally obtained as the shaker was excited by the sine signals at selected frequencies covering a usable area. The frequency response curve in the form of a deformation 


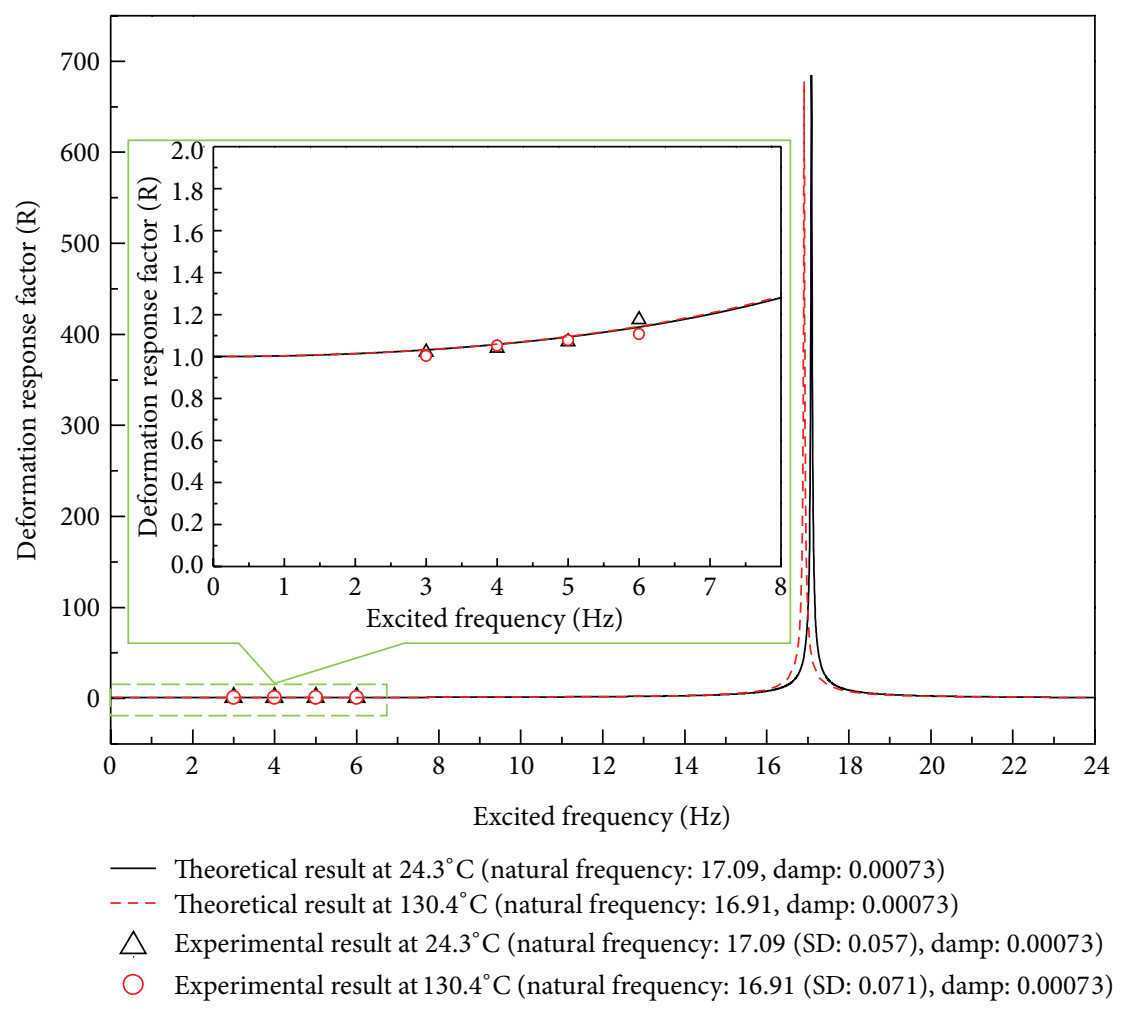

FIGURE 7: Comparison between the measured and theoretical deformation response factor $(R)$.

response factor $(R)$ was plotted as a function of the excited frequencies, as shown in Figure 7. The frequencies of the excitation signals selected were $3.0 \mathrm{~Hz}, 4.0 \mathrm{~Hz}, 5.0 \mathrm{~Hz}$, and $6.0 \mathrm{~Hz}$ in order to determine the measurable frequency range. The experimental points, which were calculated using displacements and acceleration measured by the FOA and MEMS accelerometer, respectively, were compared with the theoretical result, which was plotted using (3). The theoretical curves were plotted at $24.3^{\circ} \mathrm{C}$ and $130.4^{\circ} \mathrm{C}$, respectively, based on the assumption of negligible variation of the damping ratio at $130^{\circ} \mathrm{C}$. These theoretical curves agree very well with the experimental points measured at $24.3^{\circ} \mathrm{C}$ and $130.4^{\circ} \mathrm{C}$. The deformation response factor of the developed FOA was almost 1 in the frequency ranges below $5.1 \mathrm{~Hz}$. This implies that the designed and fabricated FOA can be used in the frequency ranges below $5.1 \mathrm{~Hz}$ within a $10 \%$ error margin in order to measure the low frequency acceleration of civil engineering structures.

\section{Conclusion}

The high temperature endurable fiber optic accelerometer was fabricated using invar, ceramic adhesive, and a $130^{\circ} \mathrm{C}$ endurable fiber optic collimator. A simple performance test setup of the accelerometer at high temperature was proposed. The developed fiber optic accelerometer measured low frequency acceleration below $5.1 \mathrm{~Hz}$ within a $10 \%$ error margin in a surrounding high temperature of $130^{\circ} \mathrm{C}$. Furthermore, the proposed fiber optic accelerometer has a very high sensitivity of $0.18\left(\mathrm{~m} / \mathrm{s}^{2}\right) / \mathrm{rad}$ at $130^{\circ} \mathrm{C}$. The fabricated sensor probe can be used for the $130^{\circ} \mathrm{C}$ endurable fiber optic accelerometer in equilibrium temperature states although the fabricated sensor in our paper may not be appropriate for steadily increasing temperature environments like most accelerometers due to sensitivity variation. For use in high temperature environments, the natural frequency change rate should be considered at the targeted temperature due to natural frequency reduction that occurs with an increase in temperature. If a leaf spring with high stiffness is used, the maximum measurable frequency range can be extended at a higher temperature. However, although the measurable frequency range would increase with greater stiffness of the leaf spring, the sensor sensitivity decreases. Therefore, it is desirable to design for a spring stiffness value appropriate for the target temperature range taking into consideration the tradeoff between sensitivity and maximum measurable frequency range. In addition, if the operating temperature limitation of the commercially available fiber optic collimator is overcome or the aimed temperature endurable fiber optic collimator with a lensed tip is developed, the developed fiber optic accelerometer can be applied to higher temperature although this research was limited to developing a $130^{\circ} \mathrm{C}$ endurable fiber optic accelerometer.

\section{Conflict of Interests}

The authors declare that there is no conflict of interests regarding the publication of this paper. 


\section{Acknowledgments}

This research was supported by the Leading Foreign Research Institute Recruitment Program (Grant no. 2011-0030065) through the National Research Foundation of Korea funded by the Ministry of Education, Science and Technology.

\section{References}

[1] B. Lee, "Review of the present status of optical fiber sensors," Optical Fiber Technology, vol. 9, no. 2, pp. 57-79, 2003.

[2] A. Wang, S. Gollapudi, K. A. Murphey, R. G. May, and R. O. Claus, "Sapphire-fiber-based intrinsic Fabry-Perot interferometer," Optics Letters, vol. 17, pp. 1021-1223, 1992.

[3] L. Tong, Y. Shen, L. Ye, and Z. Ding, "A zirconia single-crystal fibre-optic sensor for contact measurement of temperatures above $2000^{\circ} \mathrm{C}$," Measurement Science and Technology, vol. 10, no. 7, pp. 607-611, 1999.

[4] J. L. Kenndey and N. Djeu, "Operation of Yb:YAG fiber-optic temperature sensor up to $1600^{\circ} \mathrm{C}$," Sensors Actuators A, vol. 100, no. 2-3, pp. 187-191, 2002.

[5] S. Zhao, J. Zhang, C. Hou, J. Bai, and G. Yang, "Optical tilt sensor with direct intensity-modulated scheme," Optical Engineering, vol. 50, no. 11, Article ID 114405, 2011.

[6] Y. N. Kulchin, O. B. Vitrik, and A. V. Dyshlyuk, "Fiber-optic inclinometer for structural health monitoring," in Proceedings of SPIE, vol. 6662, 2007, 66620H.

[7] D. H. Kim, "A fiber-optic tiltmeter system based on the moiréfringe effect," Measurement Science and Technology, vol. 20, no. 2, Article ID 025203, 2009.

[8] M. Q. Feng and D. H. Kim, "Novel fiber optic accelerometer system using geometric moiré fringe," Sensors and Actuators A, vol. 128, no. 1, pp. 37-42, 2006.

[9] W. B. Spillman Jr., "Multimode fiber-optic hydrophone based on a schlieren technique," Applied Optics, vol. 20, no. 3, pp. 465470, 1981.

[10] S. Zhao, C. Hou, J. Bai, G. Yang, and F. Tian, "Nanometerscale displacement sensor based on phase-sensitive diffraction grating," Applied Optics, vol. 50, no. 10, pp. 1413-1416, 2011.

[11] E. Udd and P. M. Turek, "single mode fiber optic vibration sensor," in Proceedings of SPIE, vol. 566, pp. 135-140, 1985.

[12] Y. G. Lee, D. H. Kim, and C. G. Kim, "Transmissive gratingreflective mirror based fiber optic accelerometer for stable signal acquisition in industrial applications," Optical Engineering, vol. 51, no. 5, Article ID 054402, 2012.

[13] Y. G. Lee, D. H. Kim, and C. G. Kim, "Performance of a single reflective grating-based fiber optic accelerometer," Measurement Science and Technology, vol. 23, no. 4, Article ID 045101, 2012.

[14] W. Pulliam, P. Russler, R. Mlcak, K. Murphy, and C. Kozikowski, "Micromachined, SiC fiber optic pressure sensors for hightemperature aerospace applications," in Proceedings of SPIE Industrial Sensing Systems, pp. 21-30, 2000.

[15] Y. Zhu, Miniature Fiber-Optic Sensors for High-Temperature Harsh Environments [Ph.D. thesis], Virginia Polytechnic Institute and State University, 2007.

[16] Y. G. Lee, Y. Y. Kim, and C. G. Kim, "Fiber optic displacement sensor with a large extendable measurement range while maintaining equally high sensitivity, linearity, and accuracy," Review of Scientific Instruments, vol. 83, no. 4, Article ID 045002, 2012.
[17] Y. G. Lee, D. H. Kim, and C. G. Kim, "Design of patterned leaf spring for sensor-probe with stable reflectivity and high sensitivity," Sensors and Actuators A, vol. 176, pp. 19-26, 2012.

[18] J. P. Andrews, "The variation of Young's Modulus at high temperatures," Proceedings of the Physical Society of London, vol. 37, no. 1, pp. 169-177, 1924. 

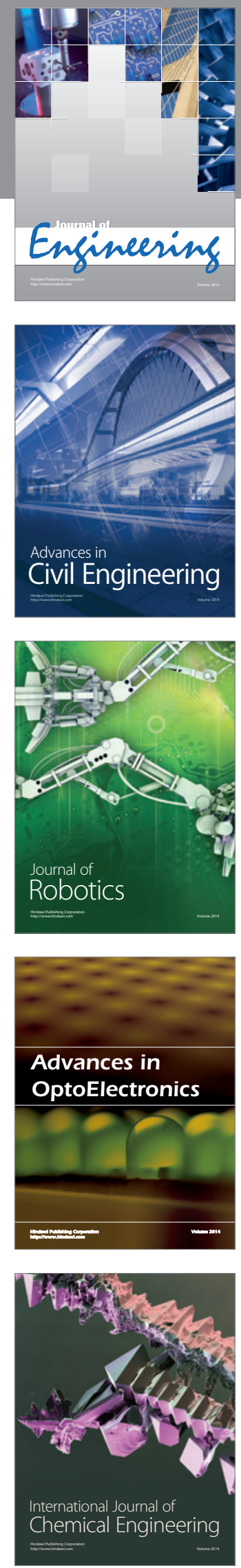

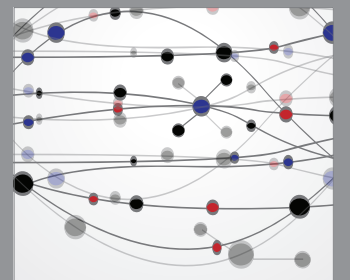

The Scientific World Journal
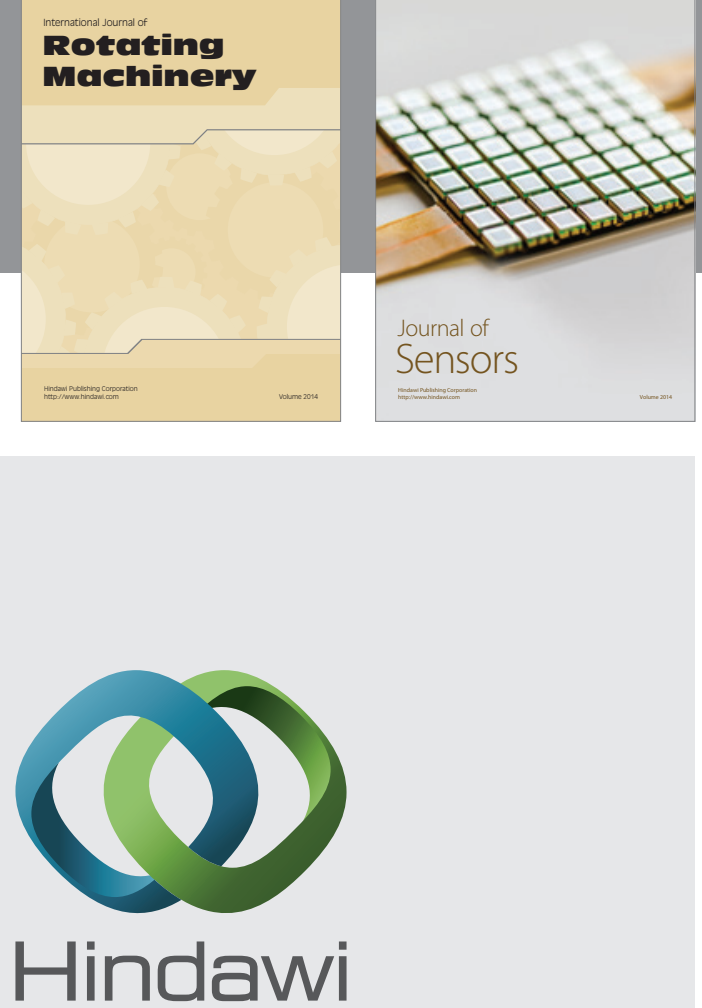

Submit your manuscripts at http://www.hindawi.com
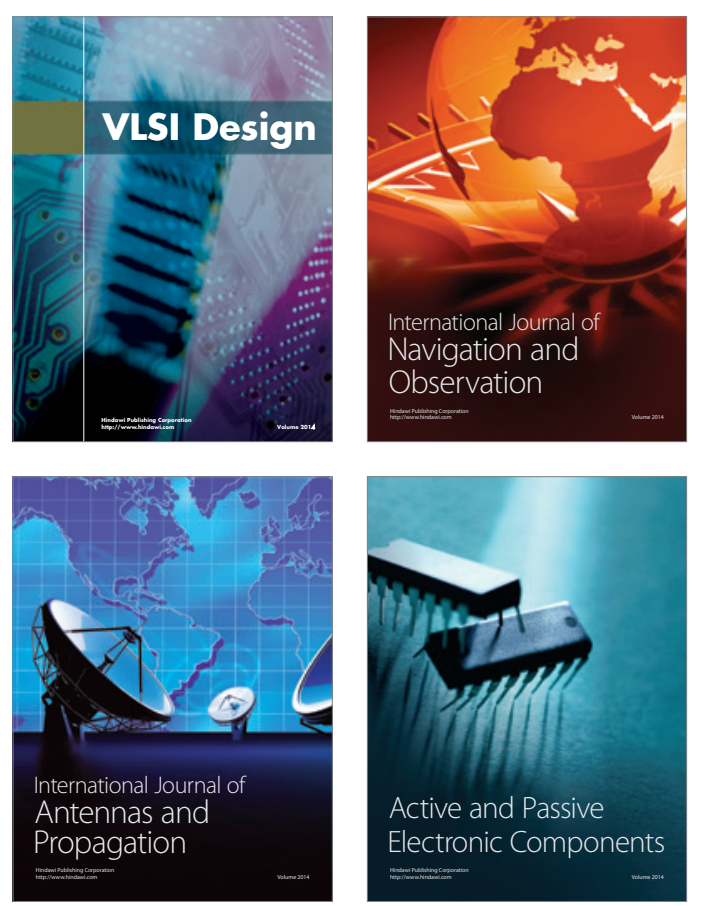
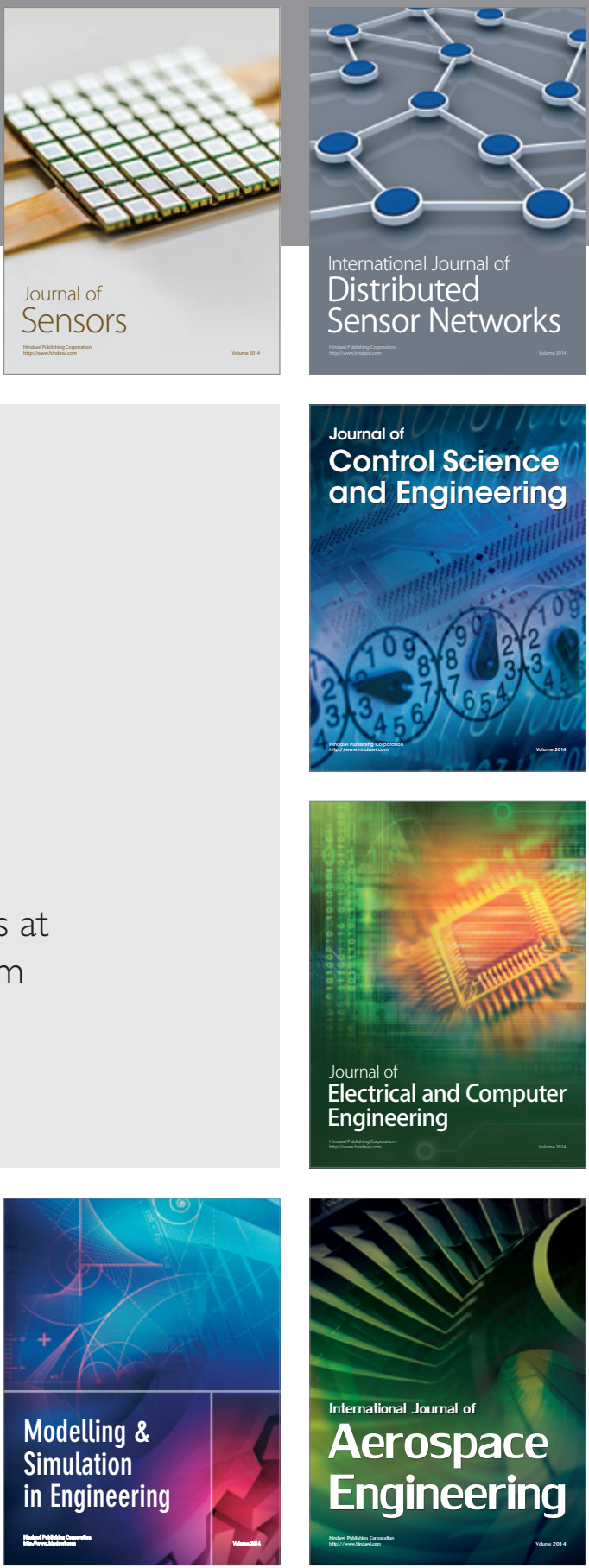

Journal of

Control Science

and Engineering
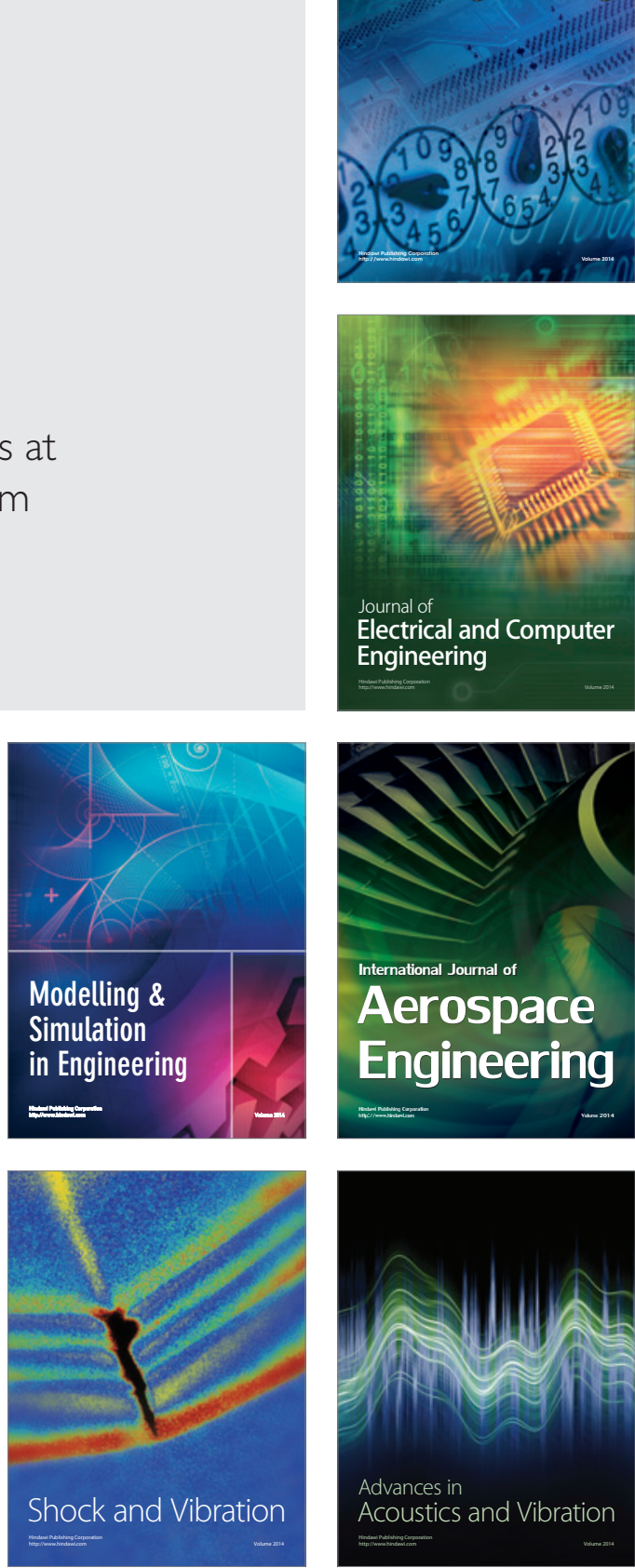